\title{
Time Fractional Generalized Korteweg-de Vries Equation: Explicit Series Solutions and Exact Solutions
}

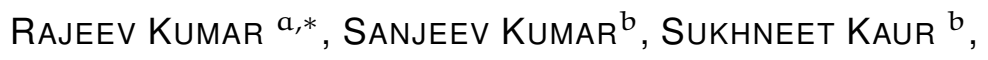 \\ SHRISHTY JAIN ${ }^{b}$ \\ a Maharishi Markandeshwar Univesity, Mullana, Ambala-131001 (Haryana), India. \\ b Thapar University, Patiala-147001 (Punjab), India.
}

\author{
- Received: 31 July 2021 • Accepted: 16 October $2021 \quad$ • Published Online:30 December 2021
}

\begin{abstract}
In this article, an attempt is made to achieve the series solution of the time fractional generalized Korteweg-de Vries equation which leads to a conditionally convergent series solution. We have also resorted to another technique involving conversion of the given fractional partial differential equations to ordinary differential equations by using fractional complex transform. This technique is discussed separately for modified Riemann-Liouville and conformable derivatives. Convergence analysis and graphical view of the obtained solution are demonstrated in this work.
\end{abstract}

Keywords: Riemann-Liouville derivative, Modified Riemann-Liouville derivative, Conformable derivative, Fractional complex transform .

2010 MSC: 26A33, 35R11.

\section{Introduction}

Non-linear partial differential equations (PDEs) have been used extensively to model many real world problems. The investigation of fractional partial differential equations (FPDEs) and fractional ordinary differential equations (FODEs) have pulled in much consideration because of the absolute portrayal of non-linear phenomena in viscosity [1], electromagnetic [2], image and signal processing [3] and other fields of applied sciences. In real life a physical phenomena depends both on instant time as well as on the past time history $[4,5]$, which can be effectively modeled by utilizing fractional operators. A classic example of generalization of partial differential equations (PDEs) to fractional partial differential equations (FPDE) is the class of Korteweg-de Vries (KdV) equations.

KdV equations are PDEs which help to formulate a model for the evolution and interaction of waves. KdV came into existence because of the experiments conducted by John Scott

*Corresponding author: rajeevkumarbudhiraja@gmail.com 
Russell (1834) and was developed further because of research done by Lord Rayleigh, Joseph Boussinesq (1870) and Korteweg and De Vries (1895). It is utilized in continuum mechanics, plasma mechanics, etc.

Numerous methods have been implemented to solve fractional KdV equations. In Ref. [6], the author has utilized He's Variational iteration method (VIM) to acquire approximate solitary wave solutions of time fractional KdV equation. Some other techniques are $\left(G^{\prime} / G\right)$ expansion method [7], Generalized Kudryashov method [8], Adomian decomposition method (ADM) [9, 10] and ADM-Pade technique [11].

The present work aims at finding the exact solution of the following time fractional generalized Korteweg-de Vries equation (TFg KdVe):

$$
\frac{\partial^{\alpha} u}{\partial t^{\alpha}}=u_{x x x}+A u^{p} u_{x}, 0<\alpha \leqslant 1, p>0
$$

For this we have explored the method discussed in $[12,13,14]$. Its algorithm constitutes conversion of FPDE to FODE in Erdèyli-Kober sense by using Lie symmetry and then derive its series solution to find the explicit solution of the governing equation. In 2012, Sahadevan and Bakkyaraj [15], had derived the Lie point symmetries of TFg KdVe(1.1) and hence used the obtained symmetries to transform it into non-linear FODE as follows:

$$
\left(P_{\frac{3}{\alpha}}^{1-\frac{2 \alpha}{3 \mathfrak{p}}-\alpha, \alpha} g\right)(z)=g^{\prime \prime \prime}(z)+\operatorname{Ag}^{p}(z) g^{\prime}(z) .
$$

They concluded that equation (1.1) cannot be solved in general except for $\alpha=p=1$. But we have made an attempt to extend their work by finding series solution of the given FODE and hence of the governing equation (1.1).

The paper is organized as follows:

In section 2 presents preliminaries, Section 3, describe the acquired solution of FODE (1.2) under some conditions. In section 4 and section 5 , fractional complex transformation(FCT) has been deployed to acquire exact solution of TFg KdVe (1.1) considering time fractional derivative in modified Riemann-Liouville(RL) and conformable derivative sense, respectively. Moreover, convergence analysis and graphical view of developed solutions have also been included in section 4 .

\section{Preliminaries}

\section{- RL fractional derivative [16]:}

$$
D_{b}^{\alpha} h(x):= \begin{cases}\frac{1}{\Gamma(m-\alpha)} \frac{d^{m}}{d x^{m}} \int_{b}^{x}(x-y)^{m-\alpha-1} h(y) d y & ; m-1<\alpha<m, \\ \frac{d^{m}}{d x^{m}} h(x) & ; \alpha=m\end{cases}
$$

where $x$ and $b$ are real numbers; $x>b, \alpha \in \mathbb{R}^{+}$and $n \in \mathbb{N}$.

\section{- Erdèyli-Kober fractional differential operator [15]}

$$
\left(P_{\beta}^{\tau, \alpha} g\right)(z):=\prod_{j=0}^{h-1}\left(\tau+j-\frac{1}{\beta} z \frac{d}{d z}\right)\left(K_{\beta}^{\tau+\alpha, h-\alpha} g\right)(z), z>0, \beta>0, \alpha>0,
$$


where $h= \begin{cases}{[\alpha]+1,} & \alpha \notin \mathbb{N}, \\ \alpha, & \alpha \in \mathbb{N},\end{cases}$

and

$$
\left(K_{\beta}^{\tau, \alpha} g\right)(z):= \begin{cases}\frac{1}{\Gamma(\alpha)} \int_{1}^{\infty}(w-1)^{\alpha-1} \mathcal{W}^{-(\tau+\alpha)} g\left(z w^{\frac{1}{\beta}}\right) \mathrm{d} w, & \alpha>0, \\ g(z), & \alpha=0,\end{cases}
$$

is Erdèyli-Kober fractional integral operator.

- Conformable Derivative [17]

Given a function $g:(0, \infty) \rightarrow \mathbb{R}$, then the conformable derivative of $g$ of order $\alpha$ is defined by:

$$
\mathrm{T}_{\alpha}(\mathrm{g})(\mathrm{t})=\lim _{\xi \rightarrow 0} \frac{g\left(t+\xi \mathrm{t}^{1-\alpha}\right)-g(t)}{\xi}, t>0, \alpha \in(0,1) .
$$

If the conformable fractional derivative of $g$ of order $\alpha$ exists, then we say $g$ is $\alpha$ differentiable.

Now, we shall find the explicit power series solution of FODE (1.2) corresponding to TFg KdV equation (1.1), deduced by the authors [15] and obtained the exact solution of the principal time fractional equation.

\section{Power Series Solution using Erdèyli-Kober Operator}

In this section, we will present a series solution of non-linear ODE of fractional order given by equation (1.2).

Suppose the power series solution for equation (1.2) is of the form:

$$
g(z)=\sum_{n=0}^{\infty} \phi_{n} z^{n}
$$

Then, the derivatives of the solution of equation (1.2) are:

$$
\begin{aligned}
g^{\prime}(z) & =\sum_{n=0}^{\infty}(n+1) \phi_{n+1} z^{n}, \\
g^{\prime \prime}(z) & =\sum_{n=0}^{\infty}(n+1)(n+2) \phi_{n+2} z^{n},
\end{aligned}
$$

and

$$
g^{\prime \prime \prime}(z)=\sum_{n=0}^{\infty}(n+1)(n+2)(n+3) \phi_{n+3} z^{n} .
$$

In view of Erdèyli-Kober (EK) fractional differential operator definition, equation(2.2) and after substituting the values of $g(z), g^{\prime \prime}(z), g^{\prime \prime \prime}(z)$ from (3.1) and (3.2), the equation (1.2) 
reads as follows:

$$
\begin{aligned}
\sum_{n=0}^{\infty} \frac{\Gamma\left(1-\frac{2 \alpha}{3 p}-\frac{n \alpha}{3}\right)}{\Gamma\left(1-\frac{2 \alpha}{3 p}-\frac{n \alpha}{3}-\alpha\right)} \phi_{n} z^{n}=\sum_{n=0}^{\infty}(n+1)(n+2)(n+3) \phi_{n+3} z^{n} \\
+A\left(\sum_{n=0}^{\infty} \phi_{n} z^{n}\right)^{p} \sum_{n=0}^{\infty}(n+1) \phi_{n+1} z^{n}
\end{aligned}
$$

The equation (3.3) can be reduced to the following cases for various values of $p$ :

Case 1: For $p=1$ equation (3.3) reduces to the following form:

$$
\begin{aligned}
\sum_{n=0}^{\infty} \frac{\Gamma\left(1-\frac{2 \alpha}{3}-\frac{n \alpha}{3}\right)}{\Gamma\left(1-\frac{5 \alpha}{3}-\frac{n \alpha}{3}\right)} \phi_{n} z^{n}=\sum_{n=0}^{\infty}(n+1)(n & +2)(n+3) \phi_{n+3} z^{n} \\
& +A\left(\sum_{n=0}^{\infty} \phi_{n} z^{n}\right) \sum_{n=0}^{\infty}(n+1) \phi_{n+1} z^{n}
\end{aligned}
$$

On comparing constant terms, coefficient of $z, z^{2}$ and $z^{n}$ on both sides of equation (3.4), one can have the following values:

$$
\begin{gathered}
\phi_{3}=\frac{1}{1.2 .3}\left(\frac{\Gamma\left(1-\frac{2 \alpha}{3}\right)}{\Gamma\left(1-\frac{5 \alpha}{3}\right)} \phi_{0}-\mathrm{A} \phi_{0} \phi_{1}\right), \\
\phi_{4}=\frac{1}{2.3 .4}\left(\frac{\Gamma(1-\alpha)}{\Gamma(1-2 \alpha)}-\mathrm{A}\left(2 \phi_{0} \phi_{2}+\phi_{1}^{2}\right)\right),
\end{gathered}
$$

and

$$
\begin{aligned}
\phi_{n+3}=\frac{1}{(n+1)(n+2)(n+3)}\left\{\frac{\Gamma\left(1-\frac{2 \alpha}{3}-\frac{n \alpha}{3}\right)}{\Gamma\left(1-\frac{5 \alpha}{3}-\frac{n \alpha}{3}\right)} \phi_{n}\right. \\
\left.\quad-A \sum_{k=0}^{n}(n+1-k) \phi_{k} \phi_{n+1-k}\right\}, \forall n \geqslant 0,
\end{aligned}
$$

respectively.

Therefore, the power series solution of FODE (1.2) for $p=1$ is given by:

$$
\begin{array}{r}
g(z)=\phi_{0}+\phi_{1} z+\phi_{2} z^{2}+\sum_{n=0}^{\infty} \frac{1}{(n+1)(n+2)(n+3)}\left\{\frac{\Gamma\left(1-\frac{2 \alpha}{3}-\frac{n \alpha}{3}\right)}{\Gamma\left(1-\frac{5 \alpha}{3}-\frac{n \alpha}{3}\right)} \phi_{n}\right. \\
\left.-A \sum_{k=0}^{n}(n+1-k) \phi_{k} \phi_{n+1-k}\right\} z^{n+3} .
\end{array}
$$


Hence, by applying the transformation [15], $z=x \mathrm{t}^{\frac{-\alpha}{3}}, \mathrm{u}(\mathrm{x}, \mathrm{t})=\mathrm{t}^{\frac{-2 \alpha}{3}} \mathrm{~g}(\mathrm{z})$, the explicit power series solution of TFG KdV equation (1.1) is given as below:

$$
\begin{array}{r}
u(x, t)=\phi_{0} t^{\frac{-2 \alpha}{3}}+\phi_{1} x t^{-\alpha}+\phi_{2} x^{2} t^{\frac{-4 \alpha}{3}}+\sum_{n=0}^{\infty} \frac{1}{(n+1)(n+2)(n+3)}\left\{\frac{\Gamma\left(1-\frac{2 \alpha}{3}-\frac{n \alpha}{3}\right)}{\Gamma\left(1-\frac{5 \alpha}{3}-\frac{n \alpha}{3}\right)} \phi_{n}\right. \\
\left.-A \sum_{k=0}^{n}(n+1-k) \phi_{k} \phi_{n+1-k}\right\} x^{n+3} t^{\frac{-\alpha(n+5)}{3}}
\end{array}
$$

Case 2: For $p=2$ equation (3.3) reads as:

$$
\begin{array}{r}
\sum_{n=0}^{\infty}\left(\frac{\Gamma\left(1-\frac{\alpha}{3}-\frac{n \alpha}{3}\right)}{\Gamma\left(1-\frac{4 \alpha}{3}-\frac{n \alpha}{3}\right)}\right) \phi_{n} z^{n}=\sum_{n=0}^{\infty}(n+1)(n+2)(n+3) \phi_{n+3} z^{n}+ \\
A\left(\sum_{n=0}^{\infty} \phi_{n} z^{n}\right)^{2} \sum_{n=0}^{\infty}(n+1) \phi_{n+1} z^{n} .
\end{array}
$$

On comparing constant terms, coefficient of $z, z^{2}$ and $z^{n}$ on both sides of equation (3.10) and after some simplification we can have the power series solution of FODE (1.2) for $p=2$ as follows:

$$
\begin{aligned}
g(z)=\phi_{0}+\phi_{1} z+\phi_{2} z^{2}+\sum_{n=0}^{\infty} \frac{1}{(n+1)(n+2)(n+3)}\left\{\frac{\Gamma\left(1-\frac{\alpha}{3}-\frac{n \alpha}{3}\right)}{\Gamma\left(1-\frac{4 \alpha}{3}-\frac{n \alpha}{3}\right)} \phi_{n}\right. \\
\left.-A \sum_{k=0}^{n} \sum_{i=0}^{k}(n+1-k) \phi_{i} \phi_{k-i} \phi_{n+1-k}\right\} z^{n+3} .
\end{aligned}
$$

Hence, for $p=2$, by applying the Lie symmetry transformation [15], $z=x t^{\frac{-\alpha}{3}}, \mathfrak{u}(x, t)=$ $t^{\frac{-\alpha}{3}} g(z)$, the explicit power series solution of TFG KdV equation is:

$$
\begin{array}{r}
u(x, t)=\phi_{0} t^{\frac{-\alpha}{3}}+\phi_{1} x t^{-\frac{2 \alpha}{3}}+\phi_{2} x^{2} t^{-\alpha}+\sum_{n=0}^{\infty} \frac{1}{(n+1)(n+2)(n+3)}\left\{\frac{\Gamma\left(1-\frac{\alpha}{3}-\frac{n \alpha}{3}\right)}{\Gamma\left(1-\frac{4 \alpha}{3}-\frac{n \alpha}{3}\right)} \phi_{n}\right. \\
\left.-A \sum_{k=0}^{n} \sum_{i=0}^{k}(n+1-k) \phi_{i} \phi_{k-i} \phi_{n+1-k}\right\} x^{n+3} t^{\frac{-\alpha(n+4)}{3}} .
\end{array}
$$

Now, we shall find the series solution of equation(3.3) in general i.e. for any value $p>0$. First of all let us simplify $z^{n}$ term of $\left(\sum_{n=0}^{\infty} \phi_{n} z^{n}\right)^{p} \sum_{n=0}^{\infty}(n+1) \phi_{n+1}$.

General term of $\left(\sum_{n=0}^{\infty} \phi_{n} z^{n}\right)^{p} \sum_{n=0}^{\infty} \phi_{n+1}(n+1) z^{n}$ is:

$$
\begin{aligned}
& \sum_{k^{(1)}=0}^{n} \sum_{k^{(2)}=0}^{k^{(1)}} \sum_{k^{(3)}=0}^{k^{(2)}} \ldots \sum_{k^{(p-2)}}^{k^{(p-3)}} \sum_{k^{(p-1)}=0}^{k^{(p-2)}} \sum_{k^{(p)}=0}^{k^{(p-1)}}\left(n+1-k^{(1)}\right) \phi_{k^{(p)}} \phi_{k^{(p-1)}-k^{(p)}} \\
& \phi_{k^{(p-2)}-k^{(p-1)}} \ldots \phi_{k^{(3)}-k^{(4)}} \phi_{k^{(2)}-k^{(3)}} \phi_{k^{(1)}-k^{(2)}} \phi_{n+1-k^{(1)}} \text {. }
\end{aligned}
$$


Upon comparing constant terms and coefficient of $z^{n}$ of equation (3.3) on both sides, one gets

$$
\phi_{3}=\frac{1}{1.2 .3}\left\{\frac{\Gamma\left(1-\frac{2 \alpha}{p}\right)}{\Gamma\left(1-\frac{2 \alpha}{3 p}-\alpha\right)} \phi_{0}-A \phi_{0}^{p} \phi_{1}\right\},
$$

and

$$
\begin{array}{r}
\phi_{n+3}=\frac{1}{(n+1)(n+2)(n+3)}\left\{\frac{\Gamma\left(1-\frac{2 \alpha}{3 p}-\frac{n \alpha}{3}\right)}{\Gamma\left(1-\frac{2 \alpha}{3 p}-\frac{n \alpha}{3}-\alpha\right)} \phi_{n}-A \sum_{k^{(1)}=0}^{n} \sum_{k^{(2)}=0}^{k^{(1)}} \sum_{k^{(3)}=0}^{k^{(2)}}\right. \\
\ldots \sum_{k^{(p-2)}=0}^{k^{(p-3)}} \sum_{k^{(p-1)}=0}^{k^{(p-2)}} \sum_{k^{(p)}=0}^{k^{(p-1)}}\left(n+1-k^{(1)}\right) \phi_{k^{(p)}} \phi_{k^{(p-1)}-k^{(p)}} \phi_{k^{(p-2)}-k^{(p-1)}} \\
\left.\ldots \phi_{k^{(3)}-k^{(4)}} \phi_{k^{(2)}-k^{(3)}} \phi_{k^{(1)}-k^{(2)}} \phi_{n+1-k^{(1)}}\right\}, \forall n \geqslant 0,
\end{array}
$$

respectively.

By substituting the values of $\phi_{3}$ and $\phi_{n+3}$ from equations (3.14) and (3.15) in (3.1), one can have:

$$
\begin{array}{r}
g(z)=\phi_{0}+\phi_{1} z+\phi_{2} z^{2}+\sum_{n=0}^{\infty} \frac{1}{(n+1)(n+2)(n+3)}\left\{\frac{\Gamma\left(1-\frac{2 \alpha}{3 p}-\frac{n \alpha}{3}\right)}{\Gamma\left(1-\frac{2 \alpha}{3 p}-\frac{n \alpha}{3}-\alpha\right)} \phi_{n}\right. \\
-A \sum_{k^{(1)}=0}^{n} \sum_{k^{(2)}=0}^{k^{(1)}} \sum_{k^{(3)}=0}^{k^{(2)}} \ldots \sum_{k^{(p-2)}=0}^{k^{(p-3)}} \sum_{k^{(p-1)}=0}^{k^{(p-2)}} \sum_{k^{(p)}=0}^{k^{(p-1)}}\left(n+1-k^{(1)}\right) \phi_{k^{(p)}} \phi_{k^{(p-1)}-k^{(p)}} \\
\left.\phi_{k^{(p-2)}-k^{(p-1)}} \ldots \phi_{k^{(3)}-k^{(4)}} \phi_{k^{(2)}-k^{(3)}} \phi_{k^{(1)}-k^{(2)}} \phi_{n+1-k^{(1)}}\right\} z^{n+3} .
\end{array}
$$

Hence, by applying the transformation [15], $z=x t^{\frac{-\alpha}{3}}, \mathfrak{u}(x, t)=t^{\frac{-2 \alpha}{3 p}} g(z)$, the explicit power series solution of TFg KdV equation (1.1) is:

$$
\begin{aligned}
& u(x, t)=\phi_{0} t^{\frac{-2 \alpha}{3 p}}+\phi_{1} x t^{\frac{-\alpha(p+2)}{3 p}}+\phi_{2} x^{2} t^{\frac{-2 \alpha(p+1)}{3 p}}+ \\
& \sum_{n=0}^{\infty} \frac{1}{(n+1)(n+2)(n+3)}\left\{\frac{\Gamma\left(1-\frac{2 \alpha}{3 p}-\frac{n \alpha}{3}\right)}{\Gamma\left(1-\frac{2 \alpha}{3 p}-\frac{n \alpha}{3}-\alpha\right)} \phi_{n}-A \sum_{k^{(1)}=0}^{n} \sum_{k^{(2)}=0}^{k^{(1)}} \sum_{k^{(3)}=0}^{k^{(2)}}\right. \\
& \ldots \sum_{k^{(p-2)}=0}^{k^{(p-3)}} \sum_{k^{(p-1)}=0}^{k^{(p-2)}} \sum_{k^{(p)}=0}^{k^{(p-1)}}\left(n+1-k^{(1)}\right) \phi_{k^{(p)}} \phi_{k^{(p-1)}-k^{(p)}} \phi_{k^{(p-2)}-k^{(p-1)}} \\
& \left.\ldots \phi_{k^{(3)}-k^{(4)}} \phi_{k^{(2)}-k^{(3)}} \phi_{k^{(1)}-k^{(2)}} \phi_{n+1-k^{(1)}}\right\} x^{n+3} t^{\frac{-\alpha((n+3) p+2)}{3 p}} \text {. }
\end{aligned}
$$

Clearly, the above series solution diverges whenever $\left(1-\frac{2 \alpha}{3 p}-\frac{n \alpha}{3}\right)$ equals zero or negative integers as gamma function is undefined for these values. Hence, the obtained solution 
(3.17) is conditionally convergent.

In next section, an alternative method is provided to find the explicit power series solution of the equation TFg KdVe (1.1).

\section{Traveling wave solution in sense of modified Riemann-Liouville derivative}

To find the traveling wave solution of equation (4.2) in modified RL [18] sense, we apply the transformation used in $[19,20]$

$$
u(x, t)=u(\xi), \xi=k x-\frac{\lambda t^{\alpha}}{\Gamma(1+\alpha)}
$$

where $k, \alpha$ are constants.

Thus, (1.1) reduces to

$$
\lambda u^{\prime}(\xi)+k^{3} u^{\prime \prime \prime}(\xi)+A k u^{p}(\xi) u^{\prime}(\xi)=0 .
$$

Integrating both sides of (4.2) with respect to $\xi$, we get

$$
\lambda u(\xi)+k^{3} u^{\prime \prime}(\xi)+A k \frac{(u(\xi))^{p+1}}{p+1}=c,
$$

where $c$ is an integration constant.

Suppose, the power series solution of (4.3) is of the following form:

$$
u(\xi)=\sum_{n=0}^{\infty} \gamma_{n} \xi^{n}
$$

Accordingly,

$$
\begin{gathered}
u^{\prime}(\xi)=\sum_{n=0}^{\infty}(n+1) \gamma_{n+1} \xi^{n}, \\
u^{\prime \prime}(\xi)=\sum_{n=0}^{\infty}(n+1)(n+2) \gamma_{n+2} \xi^{n} .
\end{gathered}
$$

Substituting the values of $u(\xi), u^{\prime}(\xi), u^{\prime \prime}(\xi)$ from equations (4.4), (4.5), (4.6) respectively the equation (4.3) can be rewritten as:

$$
\lambda \sum_{n=0}^{\infty} \gamma_{n} \xi^{n}+k^{3} \sum_{n=0}^{\infty}(n+1)(n+2) \gamma_{n+2} \xi^{n}+\frac{A k}{(p+1)}\left(\sum_{n=0}^{\infty} \gamma_{n} \xi^{n}\right)^{p+1}=c
$$

This is the general equation with $p>0$. The following cases can be deduced from equation (4.7).

Case 1: For $p=1$, equation (4.7) reads as:

$$
\lambda \sum_{n=0}^{\infty} \gamma_{n} \xi^{n}+k^{3} \sum_{n=0}^{\infty}(n+1)(n+2) \gamma_{n+2} \xi^{n}+\frac{A k}{2}\left(\sum_{n=0}^{\infty} \sum_{k=0}^{n} \gamma_{k} \gamma_{n-k} \xi^{n}\right)=c
$$


On comparing constant terms, coefficients of $\xi$ and $\xi^{n}$ on both sides of equation (4.8), one can obtain:

$$
\begin{aligned}
& \gamma_{2}=\frac{-1}{(1.2) k^{3}}\left(\frac{A k}{2} \gamma_{0}^{2}+\lambda \gamma_{0}-c\right), \\
& \gamma_{3}=\frac{-1}{(2.3) k^{3}}\left(\operatorname{Ak}\left(\gamma_{0} \gamma_{1}\right)+\lambda \gamma_{1}\right),
\end{aligned}
$$

and

$$
\gamma_{n+2}=\frac{-1}{(n+1)(n+2) k^{3}}\left(\frac{A k}{2} \sum_{k=0}^{n} \gamma_{k} \gamma_{n-k}+\lambda \gamma_{n}\right), \forall n \geqslant 1,
$$

respectively.

Thus, the power series solution of (4.3) for $p=1$ can be written as:

$$
\begin{aligned}
& u(\xi)=\gamma_{0}+\gamma_{1} \xi-\frac{1}{(1.2) k^{3}}\left(\frac{A k}{2} \gamma_{0}^{2}+\lambda \gamma_{0}-c\right) \xi^{2} \\
& \quad-\sum_{n=1}^{\infty} \frac{1}{(n+1)(n+2) k^{3}}\left(\frac{A k}{2} \sum_{k=0}^{n} \gamma_{k} \gamma_{n-k}+\lambda \gamma_{n}\right) \xi^{n+2}
\end{aligned}
$$

Hence, in view of (4.1) the exact power series solution of TFg KdV equation (1.1), for $p=1$ is defined as:

$$
\begin{aligned}
u(x, t) & =\gamma_{0}+\gamma_{1}\left(k x-\frac{\lambda t^{\alpha}}{\Gamma(1+\alpha)}\right)-\frac{1}{(1.2) k^{3}}\left(\frac{A k}{2} \gamma_{0}^{2}+\lambda \gamma_{0}-c\right)\left(k x-\frac{\lambda t^{\alpha}}{\Gamma(1+\alpha)}\right)^{2} \\
& -\sum_{n=1}^{\infty} \frac{1}{(n+1)(n+2) k^{3}}\left(\frac{A k}{2} \sum_{k=0}^{n} \gamma_{k} \gamma_{n-k}+\lambda \gamma_{n}\right)\left(k x-\frac{\lambda t^{\alpha}}{\Gamma(1+\alpha)}\right)^{n+2},
\end{aligned}
$$

where $\gamma_{0}, \gamma_{1}, k, \lambda \neq 0$ are arbitrary constants and one can obtain the remaining coefficients $\gamma_{n}(n \geqslant 1)$ from equations (4.9) and (4.11).

Case 2: For $p=2$, in equation (4.7), one can have:

$$
\lambda \sum_{n=0}^{\infty} \gamma_{n} \xi^{n}+k^{3} \sum_{n=0}^{\infty}(n+1)(n+2) \gamma_{n+2} \xi^{n}+\frac{A k}{3}\left(\sum_{n=0}^{\infty} \sum_{k=0}^{n} \sum_{i=0}^{k} \gamma_{i} \gamma_{k-i} \gamma_{n-k} \xi^{n}\right)=c
$$

On comparing constant terms, coefficients of $\xi$ and $\xi^{n}$ on both sides of equation (4.14) and after some simplification as done in previous case, one can have the exact power series solution of TFg KdVE (4.3) for $p=2$ as follows:

$$
\begin{aligned}
u(\xi)=\gamma_{0}+\gamma_{1} \xi & -\frac{1}{(1.2)} k^{3}\left(\frac{A k}{3} \gamma_{0}^{3}+\lambda \gamma_{0}-c\right) \xi^{2} \\
& -\sum_{n=1}^{\infty} \frac{1}{(n+1)(n+2) k^{3}}\left(\frac{A k}{3} \sum_{k=0}^{n} \sum_{i=0}^{k} \gamma_{i} \gamma_{k-i} \gamma_{n-k}+\lambda \gamma_{n}\right) \xi^{n+2}
\end{aligned}
$$


Therefore, the exact power series solution of TFG KdV equation (1.1) for $p=2$ is:

$$
\begin{gathered}
u(x, t)=\gamma_{0}+\gamma_{1}\left(k x-\frac{\lambda t^{\alpha}}{\Gamma(1+\alpha)}\right)-\frac{1}{(1.2)} k^{3}\left(\frac{A k}{3} \gamma_{0}^{3}+\lambda \gamma_{0}-c\right)\left(k x-\frac{\lambda t^{\alpha}}{\Gamma(1+\alpha)}\right)^{2} \\
-\sum_{n=1}^{\infty} \frac{1}{(n+1)(n+2) k^{3}}\left(\frac{A k}{3} \sum_{k=0}^{n} \sum_{i=0}^{k} \gamma_{i} \gamma_{k-i} \gamma_{n-k}+\lambda \gamma_{n}\right)\left(k x-\frac{\lambda t^{\alpha}}{\Gamma(1+\alpha)}\right)^{n+2}
\end{gathered}
$$

where $\gamma_{0}, \gamma_{1}, k, \lambda \neq 0$ are arbitrary constants.

Now, we shall find series solution of equation (4.7) in general i.e. for any value $p>0$. Hence, reduced equation is:

$$
\begin{aligned}
& \lambda \sum_{n=0}^{\infty} \gamma_{n} \xi^{n}+k^{3} \sum_{n=0}^{\infty}(n+1)(n+2) \gamma_{n+2} \xi^{n}+\frac{A k}{p+1}\left\{\sum_{n=0}^{\infty} \sum_{k^{(1)}=0}^{n} \sum_{k^{(2)}=0}^{k^{(1)}} \sum_{k^{(3)}=0}^{k^{(2)}}\right.
\end{aligned}
$$

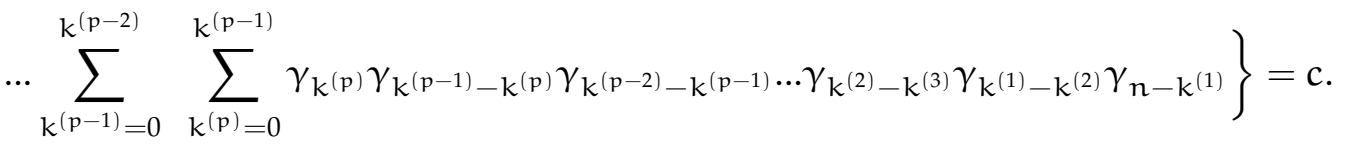

On comparing constant terms and coefficients of $\xi^{n}$ on both sides of equation (4.17), we get

$$
\gamma_{2}=\frac{-1}{(n+1)(n+2) k^{3}}\left(\frac{A k}{p+1} \gamma_{0}^{p}+\lambda \gamma_{0}-c\right)
$$

and

$$
\begin{aligned}
& \gamma_{n+2}=\frac{-1}{(n+1)(n+2) k^{3}}\left\{\frac{A k}{p+1} \sum_{k^{(1)}=0}^{n} \sum_{k^{(2)}=0}^{k^{(1)}} \sum_{k^{(3)}=0}^{k^{(2)}} \ldots \sum_{k^{(p-1)}=0}^{k^{(p-2)}} \sum_{k^{(p)}=0}^{k^{(p-1)}} \gamma_{k^{(p)}}\right. \\
& \left.\gamma_{k^{(p-1)}-k^{(p)}} \gamma_{k^{(p-2)}-k^{(p-1)} \ldots} \gamma_{k^{(2)}-k^{(3)}} \gamma_{k^{(1)}-k^{(2)}} \gamma_{n-k^{(1)}}+\lambda \gamma_{n}\right\}, \forall n \geqslant 1,
\end{aligned}
$$

respectively.

Therefore, the general solution of (4.3) is:

$$
\begin{aligned}
& u(\xi)=\gamma_{0}+\gamma_{1} \xi-\frac{1}{(n+1)(n+2) k^{3}}\left(\frac{A k}{p+1} \gamma_{0}^{p}+\lambda \gamma_{0}-c\right) \xi^{2} \\
& -\sum_{n=1}^{\infty} \frac{1}{(n+1)(n+2) k^{3}}\left\{\frac{A k}{p+1} \sum_{k^{(1)}=0}^{n} \sum_{k^{(2)}=0}^{k^{(1)}} \sum_{k^{(3)}=0}^{k^{(2)}} \ldots \sum_{k^{(p-1)}=0}^{k^{(p-2)}} \sum_{k^{(p)}=0}^{k^{(p-1)}} \gamma_{k^{(p)}}\right.
\end{aligned}
$$

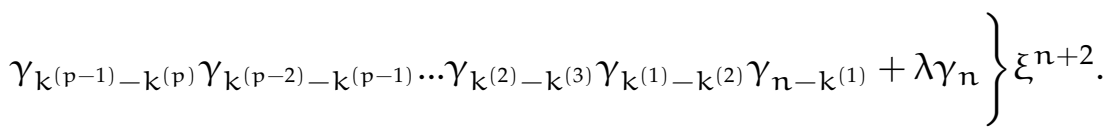


So, the exact power series solution of TFg KdVe (1.1) for any value $p>0$ is:

$$
\begin{aligned}
& u(x, t)=\gamma_{0}+\gamma_{1}\left(k x-\frac{\lambda t^{\alpha}}{\Gamma(1+\alpha)}\right) \\
& -\frac{1}{(n+1)(n+2) k^{3}}\left(\frac{A k}{p+1} \gamma_{0}^{p}+\lambda \gamma_{0}-c\right)\left(k x-\frac{\lambda t^{\alpha}}{\Gamma(1+\alpha)}\right)^{2} \\
& -\sum_{n=1}^{\infty} \frac{1}{(n+1)(n+2) k^{3}}\left\{\frac{A k}{p+1} \sum_{k^{(1)}=0}^{n} \sum_{k^{(2)}=0}^{k^{(1)}} \sum_{k^{(3)}=0}^{k^{(2)}} \ldots \sum_{k^{(p-1)}=0}^{k^{(p-2)}} \sum_{k^{(p)}=0}^{k^{(p-1)}} \gamma_{k^{(p)}}\right.
\end{aligned}
$$

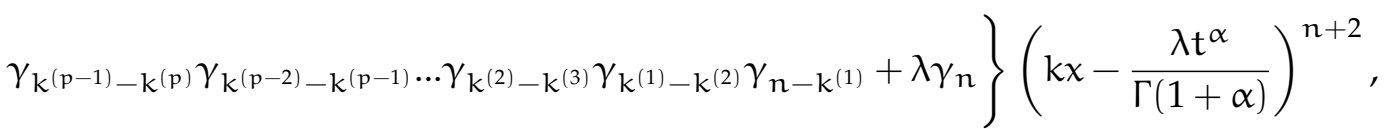

where $\gamma_{0}, k, \lambda \neq 0$ are arbitrary constants and the remaining coefficients $\gamma_{n}(n \geqslant 1)$ are obtained from equations (4.18) and (4.19).

Let us discuss the convergence analysis of series solution of equation (1.1).

\subsection{Convergence Analysis}

In this sub-section the convergence of power series solution of equation (1.1) has been analyzed by using the concepts of Implicit Function theorem [21] and majorant series. For this consider equation (4.19)

$$
\begin{aligned}
& \left|\gamma_{n+2}\right|=\mid \frac{-1}{(n+1)(n+2) k^{3}}\left\{\frac{A k}{p+1} \sum_{k^{(1)}=0}^{n} \sum_{k^{(2)}=0}^{k^{(1)}} \sum_{k^{(3)}=0}^{k^{(2)}} \ldots \sum_{k^{(p-1)}=0}^{k^{(p-2)}} \sum_{k^{(p)}=0}^{k^{(p-1)}} \gamma_{k^{(p)}}\right.
\end{aligned}
$$

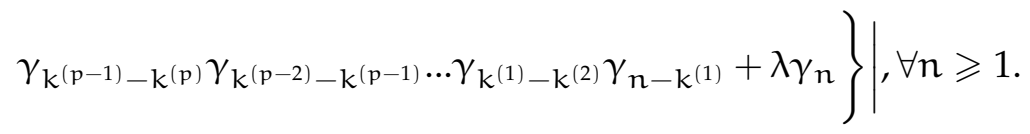

Then, one can obtain the following inequality:

$$
\begin{aligned}
& \left|\gamma_{n+2}\right| \leqslant\left|\frac{A k}{p+1}\right|\left\{\sum_{k^{(1)}=0}^{n} \sum_{k^{(2)}=0}^{k^{(1)}} \sum_{k^{(3)}=0}^{k^{(2)}} \ldots \sum_{k^{(p-1)}=0}^{k^{(p-2)}} \sum_{k^{(p)}=0}^{k^{(p-1)}}\left|\gamma_{k^{(p)}}\right|\right. \\
& \left.\left|\gamma_{k^{(p-1)}-k^{(p)}}\right|\left|\gamma_{k^{(p-2)}-k^{(p-1)}}\right| \ldots\left|\gamma_{k^{(1)}-k^{(2)}}\right|\left|\gamma_{n-k^{(1)}}\right|\right\} \\
& +|\lambda|\left|\gamma_{\mathrm{n}}\right|, \forall \mathrm{n} \geqslant 1 \text {, } \\
& \leqslant M\left[\left\{\sum_{k^{(1)}=0}^{n} \sum_{k^{(2)}=0}^{k^{(1)}} \sum_{k^{(3)}=0}^{k^{(2)}} \ldots \sum_{k^{(p-1)}=0}^{k^{(p-2)}} \sum_{k^{(p)}=0}^{k^{(p-1)}}\left|\gamma_{k^{(p)}}\right|\right.\right. \\
& \left.\left.\left|\gamma_{k^{(p-1)}-k^{(p)}}\right|\left|\gamma_{k^{(p-2)}-k^{(p-1)}}\right| \ldots\left|\gamma_{k^{(1)}-k^{(2)}}\right|\left|\gamma_{n-k^{(1)}}\right|\right\}+\left|\gamma_{n}\right|\right] \text {, }
\end{aligned}
$$


where $M=\max \left\{\left|\frac{A k}{p+1}\right|,|\lambda|\right\}$. Consider another power series,

$$
\mathrm{C}=\mathrm{C}(\xi)=\sum_{n=0}^{\infty} \mathrm{c}_{n} \xi^{n}
$$

where $c_{i}=\left|\gamma_{i}\right|$ for $i=0,1$ and

$$
\begin{aligned}
& c_{n+2}=M\left[\left\{\sum_{k^{(1)}=0}^{n} \sum_{k^{(2)}=0}^{k^{(1)}} \sum_{k^{(3)}=0}^{k^{(2)}} \ldots \sum_{k^{(p-1)}}^{k^{(p-2)}} \sum_{k^{(p)}=0}^{k^{(p-1)}} c_{k^{(p)}}\right.\right.
\end{aligned}
$$

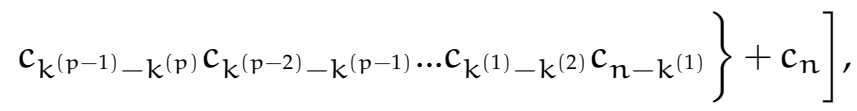

where $n=0,1,2 \ldots$

Therefore, one can deduce that:

$$
\left|\gamma_{i}\right| \leqslant c_{i}, \quad i=0,1,2 \ldots
$$

It can be easily interpreted that the series $C=C(\xi)=\sum_{n=0}^{\infty} c_{n} \xi^{n}$ is a majorant series of (4.4).

The next task is to prove that the series $C=C(\xi)$ has a positive radius of convergence. For this one may rewrite the majorant series in the following form:

$\mathrm{C}(\xi)=c_{0}+c_{1} \xi+\sum_{n=0}^{\infty} c_{n+2} \xi^{n+2}$,

$$
\begin{aligned}
& =c_{0}+c_{1} \xi+\sum_{n=0}^{\infty} M\left[\left\{\sum_{k^{(1)}=0}^{n} \sum_{k^{(2)}=0}^{k^{(1)}} \sum_{k^{(3)}=0}^{k^{(2)}} \ldots \sum_{k^{(p-1)}}^{k^{(p-2)}} \sum_{k^{(p)}=0}^{k^{(p-1)}} c_{k^{(p)}} c_{k^{(p-1)}-k^{(p)}}\right.\right.
\end{aligned}
$$

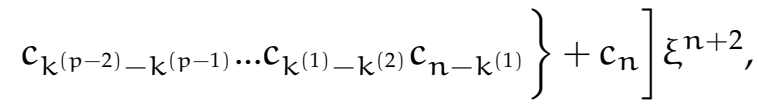

$$
\begin{aligned}
& =\mathrm{c}_{0}+\mathrm{c}_{1} \xi+M \mathrm{C}^{(\mathrm{p}+1)} \xi^{2}+M \mathrm{C}^{2} .
\end{aligned}
$$

Now, take into consideration the following implicit functional equation with $\xi$ as an independent variable:

$$
\mathrm{F}(\xi, \mathrm{C})=\mathrm{C}-\mathrm{c}_{0}-\mathrm{c}_{1} \xi-\mathrm{M}\left[\mathrm{C}^{(\mathrm{p}+1)} \xi^{2}+\mathrm{C} \xi^{2}\right] .
$$

From the above equation, we observe that $F$ is analytical in the neighborhood of $\left(0, c_{0}\right)$ and $\mathrm{F}\left(0, \mathrm{c}_{0}\right)=0 ; \mathrm{F}_{\mathrm{C}}^{\prime}\left(0, \mathrm{c}_{0}\right)=1 \neq 0$.

Then by Implicit Function theorem [21], it can be inferred that $C=C(\xi)$ is analytic in the neighborhood of the point $\left(0, c_{0}\right)$ with positive radius of convergence.

Hence, the power series (4.4) is convergent in neighborhood of the point $\left(0, c_{0}\right)$. 


\subsection{Graphical Report}

The figures of explicit solutions of TFg KdV equation (1.1), obtained using FCT and power series method have been presented.
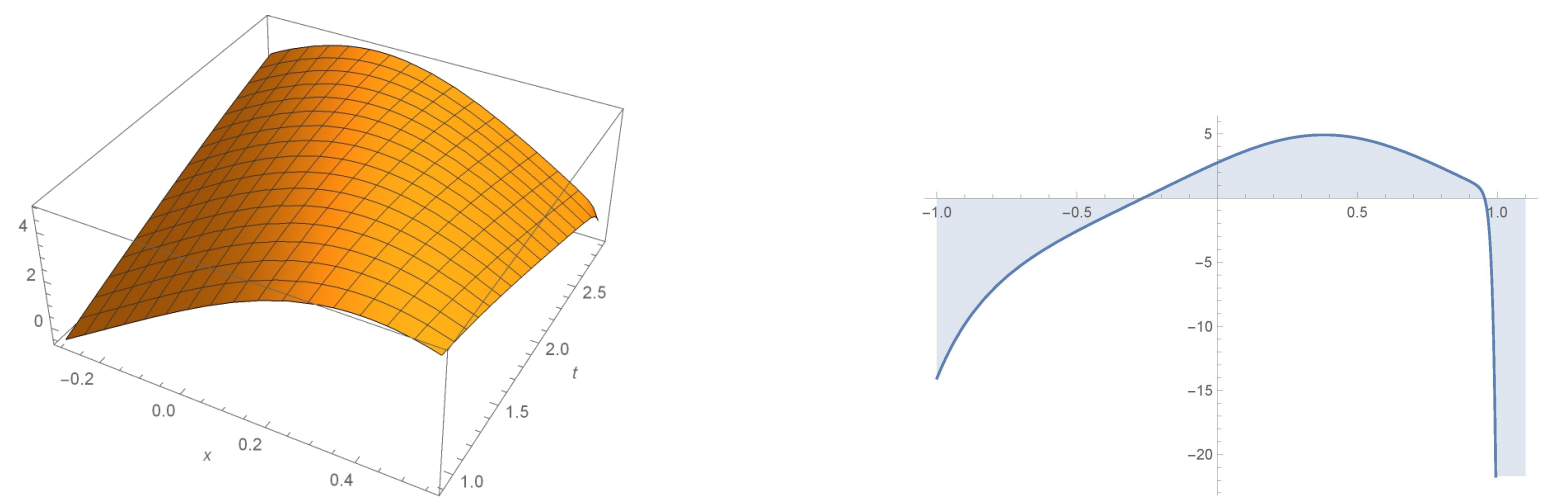

Figure 1: Perspective and 2D view of power series solution of (4.13) with the parameter values $\gamma_{0}=0, \gamma_{1}=$ $\frac{32}{3}, \alpha=.85, k=1, A=3, \lambda=\frac{-1}{4}$.
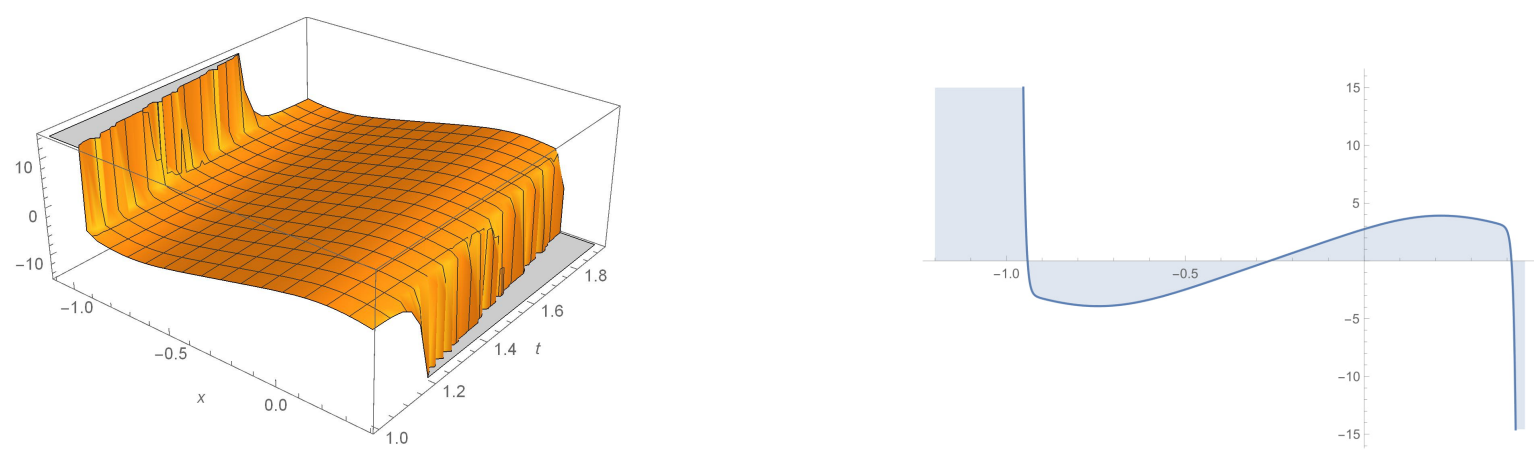

Figure 2: Perspective and 2D view of explicit series solution of (4.16) with the parameter values $\gamma_{0}=0, \gamma_{1}=$ $\frac{32}{3}, \alpha=.85, k=1, A=3, \lambda=\frac{-1}{4}$. 


\section{Traveling Wave Solution in Sense of Conformable Derivative}

This section aims at finding the solution of TFg KdVe (1.1), where time fractional derivative of order $\alpha$ is considered as conformable derivative (2.4). The reduced ODE is solved by using $\left(\mathrm{G}^{\prime} / \mathrm{G}\right)$ expansion method $[22,23]$. In light of the following transformation:

$$
u=f(\xi), \xi=x+\frac{k t^{\alpha}}{\alpha},
$$

the equation (1.1) reduces into an ordinary differential equation of the following form:

$$
f^{\prime \prime \prime}(\xi)-k f^{\prime}(\xi)+A f^{p}(\xi) f(\xi)=0 .
$$

On integrating and taking integration constant equal to zero, (5.2) reduces to:

$$
f^{\prime \prime}(\xi)-k f(\xi)+A \frac{(f(\xi))^{p+1}}{(p+1)}=0 .
$$

Consider the following three cases for equation (5.3)

Case 1: when $p=1$

Then, (5.3) gets converted to:

$$
f^{\prime \prime}(\xi)-k f(\xi)+A \frac{f(\xi)^{2}}{2}=0 .
$$

By homogeneous balance method, one can obtain degree $n=2$. Hence, by utilizing $\left(\frac{\mathrm{G}^{\prime}}{\mathrm{G}}\right)$ method, suppose solution is of the following form:

$$
f(\xi)=w_{0}+w_{1}\left(\frac{G^{\prime}}{G}\right)+w_{2}\left(\frac{G^{\prime}}{G}\right)^{2} .
$$

After substituting (5.5) in (5.4), a set of equations in terms of $w_{0}, w_{1}, w_{2}$ are obtained as:

$$
\begin{gathered}
20 w_{2}^{2} \lambda+10 w_{2} w_{1}+\frac{1}{2} A w_{2}^{2}=0 \\
8 w_{2}^{2} \lambda+A w_{1} w_{2}+16 w_{2}^{2} \mu+2 w_{1}^{2}+16 w_{2} \lambda w_{1}=0 \\
A w_{0} w_{2}+12 w_{2}^{2} \lambda \mu+\frac{1}{2} A w_{1}^{2}+12 w_{2} w_{1} \mu+3 w_{1}^{2} \lambda-k w_{2}+6 w_{1} \lambda^{2} w_{2}=0 \\
w_{1}^{2} \lambda^{2}+2 w_{1}^{2} \mu+A w_{0}-k w_{1}+8 w_{1} \lambda w_{2} \mu+4 w_{2}^{2} \mu^{2}=0 \\
w_{1}^{2} \lambda \mu+2 w_{2} \mu^{2} w_{1}+\frac{1}{2} A w_{0}^{2}-k w_{0}=0 \\
12 w_{2}^{2}=0 .
\end{gathered}
$$


On solving, it gives $w_{2}=0, w_{1}=\frac{-36\left(A w_{0}-K\right)}{A^{2}+72 \mu}, w_{0}=w_{0}, \mu=\mu, \lambda=\frac{-A}{6}$. Depending on $\lambda^{2}-4 \mu$ there are two solutions.

If $\lambda^{2}-4 \mu \succ 0$, it gives hyperbolic traveling wave solution of equation (5.4) as

$$
u(x, t)=w_{0}-\frac{36\left(A w_{0}-K\right)}{A^{2}+72 \mu} \frac{\sqrt{\lambda^{2}-4 \mu}}{2}\left[\frac{\left(L_{1} \operatorname{Cosh} \frac{\sqrt{\lambda^{2}-4 \mu}}{2} \xi+L_{2} \operatorname{Sinh} \frac{\sqrt{\lambda^{2}-4 \mu}}{2} \xi\right)}{\left(L_{1} \operatorname{Sinh} \frac{\sqrt{\lambda^{2}-4 \mu}}{2} \xi+L_{2} \operatorname{Cosh} \frac{\sqrt{\lambda^{2}-4 \mu}}{2} \xi\right)}-\frac{\lambda}{2}\right],
$$

where $\xi=x+\frac{k t^{\alpha}}{\alpha}$.

If $\lambda^{2}-4 \mu \prec 0$, it gives trigonometric traveling wave solution of equation (5.4) as

$$
u(x, t)=w_{0}-\frac{36\left(A w_{0}-K\right)}{A^{2}+72 \mu} \frac{\sqrt{4 \mu-\lambda^{2}}}{2}\left[\frac{\left(L_{1} \operatorname{Cos} \frac{\sqrt{4 \mu-\lambda^{2}}}{2} \xi-L_{2} \operatorname{Sin} \frac{\sqrt{4 \mu-\lambda^{2}}}{2} \xi\right)}{\left(L_{1} \operatorname{Sin} \frac{\sqrt{4 \mu-\lambda^{2}}}{2} \xi+L_{2} \operatorname{Cos} \frac{\sqrt{4 \mu-\lambda^{2}}}{2} \xi\right)}-\frac{\lambda}{2}\right],
$$

where $\xi=x+\frac{k t^{\alpha}}{\alpha}$.

Case 2: when $p=2$

Then, (5.3) reduces to:

$$
f^{\prime \prime}(\xi)-k f(\xi)+A \frac{\{f(\xi)\}^{3}}{3}=0 .
$$

By homogeneous balance method, one can obtain degree $n=1$. Hence, by using $\left(\frac{\mathrm{G}^{\prime}}{\mathrm{G}}\right)$ method, suppose solution is of the following form:

$$
f(\xi)=w_{0}+w_{1}\left(\frac{G^{\prime}}{G}\right) .
$$

Putting (5.10) in equation (5.9) gives a set of equations in terms of $w_{0}, w_{1}$, on simplifying it , $w_{1}=-6 \frac{1}{\sqrt{-6 \mathrm{~A}}}, w_{0}=-3 \frac{\lambda}{\sqrt{-6 \mathrm{~A}}}, \mu=\frac{1}{4} \lambda^{2}+\frac{\mathrm{k}}{2}$

If $\lambda^{2}-4 \mu \succ 0$

$$
u(x, t)=-3 \frac{\lambda}{\sqrt{-6 A}}-6 \frac{1}{\sqrt{-6 A}}\left[\frac{\left(L_{1} \operatorname{Cosh} \frac{\sqrt{\lambda^{2}-4 \mu}}{2} \xi+L_{2} \operatorname{Sinh} \frac{\sqrt{\lambda^{2}-4 \mu}}{2} \xi\right)}{\left(L_{1} \operatorname{Sinh} \frac{\sqrt{\lambda^{2}-4 \mu}}{2} \xi+L_{2} \operatorname{Cosh} \frac{\sqrt{\lambda^{2}-4 \mu}}{2} \xi\right)}-\frac{\lambda}{2}\right]
$$

where $\xi=x+\frac{k t^{\alpha}}{\alpha}$.

If $\lambda^{2}-4 \mu \prec 0$

$$
u(x, t)=-3 \frac{\lambda}{\sqrt{-6 A}}-6 \frac{1}{\sqrt{-6 A}}\left[\frac{\left(L_{1} \operatorname{Cos} \frac{\sqrt{4 \mu-\lambda^{2}}}{2} \xi-L_{2} \operatorname{Sin} \frac{\sqrt{4 \mu-\lambda^{2}}}{2} \xi\right)}{\left(L_{1} \operatorname{Sin} \frac{\sqrt{4 \mu-\lambda^{2}}}{2} \xi+L_{2} \operatorname{Cos} \frac{\sqrt{4 \mu-\lambda^{2}}}{2} \xi\right)}-\frac{\lambda}{2}\right]
$$

where $\xi=x+\frac{k t^{\alpha}}{\alpha}$.

Case 3: For any value of $p>0$

Then, (5.3) reduces to:

$$
f^{\prime \prime}-k f+B f^{(p+1)}=0 .
$$


where $B=\frac{A}{(p+1)}$. By homogeneous balance method, one can obtain degree $n=\frac{2}{p}$. Hence, by using $\left(\frac{\mathrm{G}^{\prime}}{\mathrm{G}}\right)$ method, suppose solution is of the following form:

$$
f(\xi)=q\left(\frac{G^{\prime}}{G}\right)^{\frac{2}{p}}
$$

Putting equation (5.14) in (5.13) gives a set of equation in polynomials $\left(\frac{\mathrm{G}^{\prime}}{\mathrm{G}}\right)$ of which is used to find out constant $q$. The obtained value of $q=\left(\frac{-2 p-4}{B p^{2}}\right)^{\frac{1}{p}}, \lambda=0, \mu=\frac{k p^{2}}{8}$. The solution of equation (5.14) is given as according to the conditions on $\mu$. If $\mu \succ 0$, then

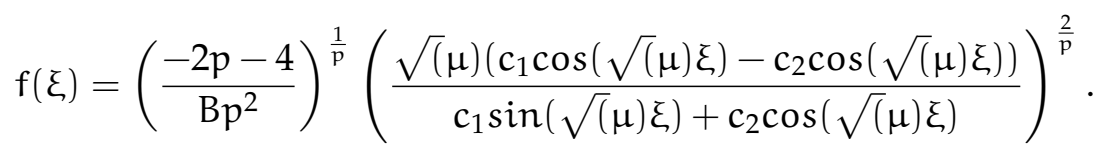

If $\mu \prec 0$, then

$$
f(\xi)=\left(\frac{-2 p-4}{B p^{2}}\right)^{\frac{1}{p}}\left(\frac{-\sqrt{(\mu)\left(c_{3} e^{\sqrt{(\mu) \xi}}-c_{4} e^{-\sqrt{(\mu) \xi})}\right.}}{c_{3} e^{\sqrt{(\mu) \xi}}+c_{4} e^{-\sqrt{(\mu) \xi}}}\right)^{\frac{2}{p}}
$$

\section{Conclusion}

Sahadevan and Bakkyaraj [15] have reduced TFg KdV equation (1.1) to FODE (1.2), by the concept of Lie symmetry. They claimed that the reduced form cannot be solved in general. But any equation without solution has no significance. Motivated by this fact, in this study not only conditionally convergent series solution has been provided, but also we have proposed one of the wave solution in sense of modified Riemann-Liouville derivative. Graphs and convergence analysis of the procured series solution have been put forth. Further, $\left(G^{\prime} / G\right)$ expansion method has been deployed to solve ODE (5.3) corresponding to TFg KdVe (1.1) in conformable derivative sense.

\section{References}

[1] Kumar D, Singh J and Kumar S (2015). A fractional model of Navier-Stokes equation arising in unsteady flow of a viscous fluid. J. Assoc. Arab Univ. Basic Appl. Sci. 17:14-19. https://doi.org/10.1016/j.jaubas.2014.01.001

[2] Das S. (2011)."Functional Fractional Calculus". Berlin. Springer, 2.https://doi.org/10.1007/978-3-64220545-3

[3] Yang Q, Chen Dali and Zhao T and Chen Y (2016), Fractional calculus in image processing: a review, Fract. Calc. Appl. Anal. 19: 1222-1249. https://doi.org/10.1515/fca-2016-0063

[4] Shah K, Seadawy AR and Arfan M.(2020), Evaluation of one dimensional fuzzy fractional partial differential equations, Alex. Eng. J., 59:3347-3353. https://doi.org/10.1016/j.aej.2020.05.003

[5] Gul H, Alrabaiah H, Ali S, Shah K and Shakoor M (2020). Computation of solution to fractional order partial reaction diffusion equations. J. Adv. Res. 25:31-38. https://doi.org/10.1016/j.jare.2020.04.021

[6] Zhang Y (2014). Formulation and solution to time-fractional generalized Korteweg-de Vries equation via variational methods. Adv. Differ. Equ.. 2014:1-12.https://doi.org/10.1186/1687-1847-2014-65 
[7] Sahoo S and Ray S (2016). Solitary wave solutions for time fractional third order modified KdV equation using two reliable techniques $\left(G^{\prime} / G\right)$-expansion method and improved $\left(G^{\prime} / G\right)$-expansion method. Phys A: Stat. Mech. App. 448:265-282. https://doi.org/10.1016/j.physa.2015.12.072

[8] Demiray TS, Pandir Yusuf and Bulut H (2020). Generalized Kudryashov method for time-fractional differential equations. Abstr. Appl. Anal., 2014: 901540 1-13. https://doi.org/10.1155/2014/901540

[9] El-Wakil, SA, Elhanbaly A and Abdou, MA (2006). Adomian decomposition method for solving fractional nonlinear differential equations. Appl. Math. and Comput. 182:313-324. https://doi.org/10.1016/j.amc.2006.02.055

[10] Xueqin Lv and Gao J (2017). Treatment for third-order nonlinear differential equations based on the Adomian decomposition method, LMS J. Comput. Math. 20:1-10. https://doi.org/10.1112/S1461157017000018

[11] Abassy T., El-Tawil MA and Saleh HK (2004). The solution of KdV and mKdV equations using Adomian Pade approximation. Int. J. Nonlinear Sci. Numer. Simul.. 5:327-339. https://doi.org/10.1515/IJNSNS.2004.5.4.327

[12] Inc M, Yusuf A, Aliyu IA and Baleanu D (2018). Lie symmetry analysis, explicit solutions and conservation laws for the space-time fractional nonlinear evolution equations. Phys A: Stat. Mech. App. 496:371-383. https://doi.org/10.1016/j.physa.2017.12.119

[13] Inc M, Yusuf A, Aliyu AI and Baleanu D (2018). Lie symmetry analysis and explicit solutions for the time fractional generalized Burgers-Huxley equation . Opt. Quantum Electron. 50:94-100. https://doi.org/10.1007/s11082-018-1373-8

[14] Inc M, Yusuf A, Aliyu AI and Baleanu D (2018). Time-fractional Cahn-Allen and time-fractional KleinGordon equations: Lie symmetry analysis, explicit solutions and convergence analysis. Phys A: Stat. Mech. App. 493: 94-106. https://doi.org/10.1016/j.physa.2017.10.010

[15] Sahadevan $\mathrm{R}$ and Bakkyaraj T(2012). Invariant analysis of time fractional generalized Burgers and Korteweg-de Vries equations, J. Math. Anal. Appl.. 393:341-347. https://doi.org/10.1016/j.jmaa.2012.04.006

[16] Podlubny, Igor(1998), "Fractional Differential Equations: An Introduction to Fractional Derivatives, Fractional Differential Equations, to Methods of Their Solution and Some of Their Applications"'. Elsevier.

[17] Khalil R, Horani MAl, Yousef A and Sababheh M (2014). A new definition of fractional derivative. J. Comput. Appl. Math. 264:65-70. https://doi.org/10.1016/j.cam.2014.01.002

[18] Jumarie G (2009). Table of some basic fractional calculus formulae derived from a modified Riemann-Liouville derivative for non-differentiable functions. Appl. Math. Lett. 22:378-385. https://doi.org/10.1016/j.aml.2008.06.003

[19] Qin CY, Tian SF, Wang XB and Zhang T (2017). Lie symmetry analysis, conservation laws and explicit solutions for the time fractional Rosenau-Haynam equation. Waves in Rand. Compl. Media. 27:308-324. https://doi.org/10.1080/17455030.2016.1231434

[20] Chauhan A and Arora R (2019). Time fractional Kupershmidt equation: symmetry analysis and explicit series solution with convergence analysis. Commun. Math. 27:171-185. https://doi.org/10.2478/cm2019-0013

[21] Rudin, Walter(1964). "Principles of Mathematical Analysis"'. New York. McGraw-hill.

[22] Cenesiz Y, Dumitru B, Kurt A and Tasbozan O(2017). New exact solutions of Burgers type equations with conformable derivative. Waves in Rand. Compl. Media. 27:103-116. https://doi.org/10.1080/17455030.2016.1205237

[23] Kurt A, Tasbozan, Oand Baleanu, Dumitru (2017). New solutions for conformable fractional NizhnikNovikov-Veselov system via $G^{\prime} / G$ expansion method and homotopy analysis methods. Opt. Quantum Electron 49333-341. https://doi.org/10.1007/s11082-017-1163-8

[24] Kumar R, Gupta RK and Bhatia SS (2016). Invariant solutions of variable coefficients generalized Gardner equation, Nonlinear Dyn., 83:2103-2111. https://doi.org/10.1007/s11071-015-2468-4 